\title{
Contribution of the t-channel $N^{*}(1535)$ exchange for the $p \bar{p} \rightarrow \phi \phi$ reaction
}

\author{
Jun Shi ${ }^{1,2}$, Jian-Ping Dai ${ }^{1}$, and Bing-Song Zou ${ }^{1,3}$ \\ ${ }^{1}$ Institute of High Energy Physics and Theoretical Physics \\ Center for Sciences Facilities, CAS, Beijing 100049, China \\ 2 Shandong university at Weihai, Weihai 264209, China \\ ${ }^{3}$ Center of Theoretical Nuclear Physics, \\ National Laboratory of Heavy Ion Collisions, Lanzhou 730000, China
}

\begin{abstract}
Since the $N^{*}(1535)$ resonance was found to have large coupling to the strangeness due to its possible large $s \bar{s}$ component, we investigate the possible contribution of the t-channel $N^{*}(1535)$ exchange for the $p \bar{p} \rightarrow \phi \phi$ reaction. Our calculation indicates that the new mechanism gives very significant contribution for the energies above $2.25 \mathrm{GeV}$ and may be an important source for evading the Okubo-Zweig-Iizuka rule in the $\phi$ production from $N \bar{N}$ annihilation.

PACS numbers: 13.75.-n, 13.75.Cs, 14.20.Gk, 25.75.Dw
\end{abstract}




\section{INTRODUCTION}

$\phi$ meson production from $N \bar{N}$ annihilation has been drawing intensive interest for its violation of the Okubo-Zweig-Iizuka (OZI) rule [1 3]. The usual statement of the OZI rule is that diagrams with disconnected quark lines can be negligible compared to those with connected quark lines. $\phi$ meson is believed to be an almost pure $s \bar{s}$ state, while nucleon is universally considered to be composed of up and down quarks. Thus according to the OZI rule, the reaction that $N \bar{N}$ annihilate to produce $\phi$ is to be suppressed. However, it is known that $\phi$ production evades the OZI rule in various hadronic reactions [4]. The study of the reaction is seen as a promising probe into the strangeness information of nucleon or nucleon resonances.

To interpret the substantial OZI rule violations, Lindenbaum [5] and Etkin et al. [6] pointed out the intervention of glueball resonances, Dover [7] argued $s \bar{s} n \bar{n}$ four quark states produce selective enhancements, Kochelev [8] took instanton effects into account, Ellis et al. [9, 10] inferred considerable admixture of $s \bar{s}$ components in the nucleon. Also, as OZIforbidden reactions can proceed via two-step hadronic loops in which each individual transition is OZI-allowed [11], Lu et al. [12] studied two-meson $(K \bar{K})$ intermediate states and Mull et al. [13] investigated antihyperon-hyperon $(\Lambda \bar{\Lambda})$ intermediate states, both of them educed compatible theoretical results with the experimental data.

On the other hand, recently, it has been suggested that the strong couplings of $N^{*}(1535)$ to $\eta N, \eta^{\prime} N$ and $K \Lambda$ may indicate a possible significant $s \bar{s}$ component in the quark wave function of $N^{*}$ (1535) and also a large coupling to $\phi N$ [14 16]. Xie et al. [14] deduced the significant coupling of $N^{*}(1535)$ to $N \phi$ as $g_{N^{*}(1535) N_{\phi}}^{2} / 4 \pi=0.13$. With such effective $N^{*}(1535) N \phi$ coupling, the measured $\pi^{-} p \rightarrow n \phi, p p \rightarrow p p \phi$ and $p n \rightarrow \phi d$ cross sections are well reproduced [14, 15].

In this article, we extend the model used in Ref. [14] to study the $p \bar{p} \rightarrow \phi \phi$ reaction. The scenario is that $p \bar{p}$ interact with each other by exchanging $N^{*}(1535)$ in $t$ channel, which has not been attempted by others so far. 

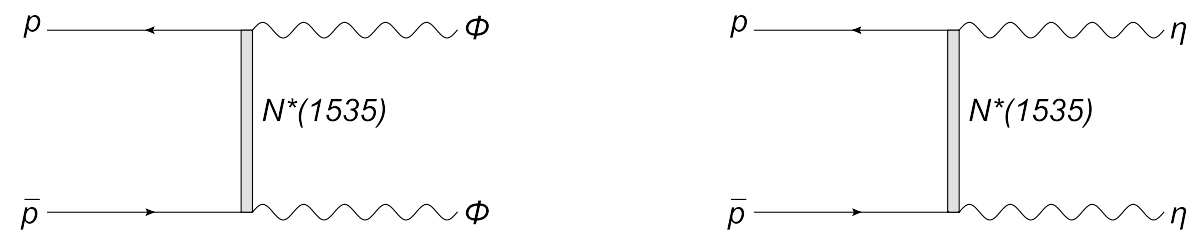

FIG. 1: Feynman diagrams for $p \bar{p} \rightarrow \phi \phi$ and $\eta \eta$ with $t$-channel $N^{*}(1535)$ exchange

\section{FORMALISM AND INGREDIENTS}

The Feynman diagram for the $p \bar{p} \rightarrow \phi \phi$ reaction is depicted in Fig.1 (left). We use the effective Lagrangian for $N^{*}(1535) N \phi$ as [14]

$$
\mathcal{L}_{\phi N N^{*}}=i g_{N^{*} N \phi} \bar{N} \gamma_{5}\left(\gamma^{\mu}-\frac{q^{\mu} \not q}{q^{2}}\right) \phi_{\mu} N^{*}+\text { h.c. }
$$

Here $N$ and $N^{*}$ are the spin wave functions for the nucleon and $N^{*}(1535)$ resonance, respectively, and $\phi_{\mu}$ is the $\phi$-meson field. For the $N^{*}(1535)-N$ - $\phi$ vertices, the following monopole form factor is used:

$$
F_{N^{*}}\left(q^{2}\right)=\frac{\Lambda^{2}-m_{N^{*}}^{2}}{\Lambda^{2}-q^{2}} .
$$

Empirically the cut-off parameter $\Lambda$ for $N^{*}(1535)$ should be at least a few hundred $\mathrm{MeV}$ larger than the $N^{*}$ mass, hence to be in the range of 2 to $4 \mathrm{GeV}$.

Then the amplitude and cross section can be obtained straightforwardly by applying the Feynman rules to Fig.1 (left).

$$
\begin{aligned}
\mathcal{M}= & -g_{N^{*} N \phi}^{2} F_{N^{*}}^{2}\left(q^{2}\right) \bar{v}_{\bar{p}}\left(p^{\prime}, s^{\prime}\right) \gamma_{5}\left(\gamma^{\mu}-\frac{q^{\mu} \not q}{q^{2}}\right) \epsilon_{\mu}^{*}\left(k^{\prime}\right) \frac{i}{\not q-M_{N^{*}}} \gamma_{5}\left(\gamma^{\nu}-\frac{q^{\nu} \not q}{q^{2}}\right) \epsilon_{\nu}^{*}(k) u_{p}(p, s) \\
& \left.+ \text { (exchange term with } k \leftrightarrow k^{\prime}\right)
\end{aligned}
$$

with $q=p-k$, and

$$
\frac{d \sigma}{d \Omega}=\frac{|\vec{k}|}{256 \pi^{2}|\vec{p}|\left(p+p^{\prime}\right)^{2}} \sum_{s, s^{\prime}}|\mathcal{M}|^{2}
$$

\section{NUMERICAL RESULTS AND DISCUSSION}

Fig. 2 shows the theoretical results for the contribution from the t-channel $N^{*}(1535)$ exchange to the $p \bar{p} \rightarrow \phi \phi$ reaction with various $\Lambda$ parameter, compared with the experimental data [17]. One can see that for the invariant mass $s^{1 / 2}$ above $2.3 \mathrm{GeV}$ the new mechanism alone reproduces the data well with $\Lambda \sim 3 \mathrm{GeV}$. 


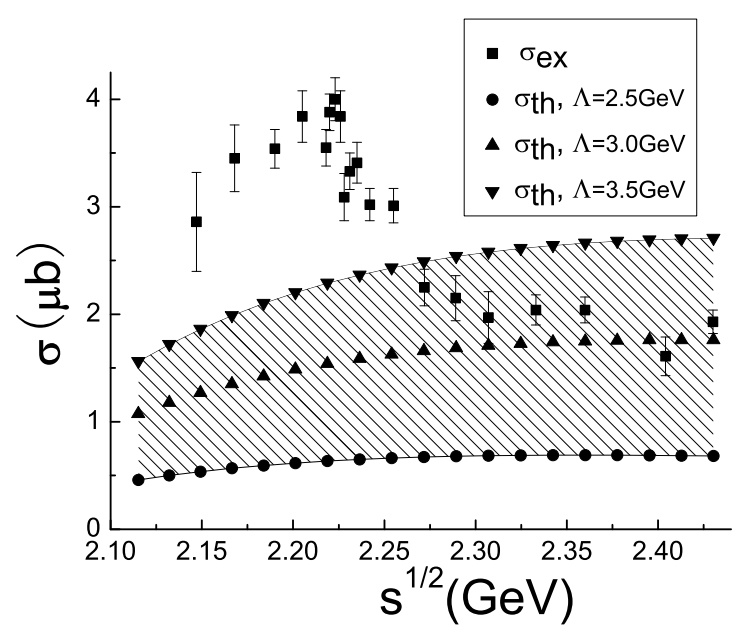

FIG. 2: Theoretical results for the contribution from the t-channel $N^{*}(1535)$ exchange to the $p \bar{p} \rightarrow \phi \phi$ reaction with various $\Lambda$ parameter, compared with data [17].

To check whether the new mechanism and its $\Lambda$ parameter are reasonable, we also use the same approach to study $p \bar{p} \rightarrow \eta \eta$ as shown in Fig.1 (right). The interaction Lagrangian for $N^{*}(1535) N \eta$ coupling is [14]:

$$
\mathcal{L}_{\eta N N^{*}(1535)}=i g_{N^{*} N \eta} \bar{N} \eta N^{*}+\text { h.c. },
$$

with $g_{\eta N N^{*}(1535)} / 4 \pi=0.28$ [14]. The theoretical results for the contribution from the tchannel $N^{*}(1535)$ exchange with $\Lambda=3$ and $3.5 \mathrm{GeV}$ are shown in Fig.3. Compared with experimental data [18], the contribution is very small. This is consistent with the partial wave analysis of Ref.[19] which suggests a large s-channel resonant contribution from $0^{++}$, $2^{++}$and $4^{++}$meson resonances with masses of $2.0 \sim 2.4 \mathrm{GeV}$. These meson resonances have strong couplings to both $p \bar{p}$ and $\eta \eta$, allowed by the OZI rule. Hence the t-channel $N^{*}(1535)$ exchange only plays a minor role for the $p \bar{p} \rightarrow \eta \eta$ reaction. These meson resonances can also decay to $\phi \phi$ through strange meson or baryon loops [12, 13], which give a few times smaller rate. Then the t-channel $N^{*}(1535)$ exchange plays a more important role for the $p \bar{p} \rightarrow \phi \phi$ reaction. However, while the contribution from the t-channel $N^{*}(1535)$ exchange can reproduce the $p \bar{p} \rightarrow \phi \phi$ cross sections above $2.3 \mathrm{GeV}$, it cannot reproduce the peak structure around $2.2 \mathrm{GeV}$. This suggests that the s-channel resonances may still dominates here through the strange meson or baryon loops. 


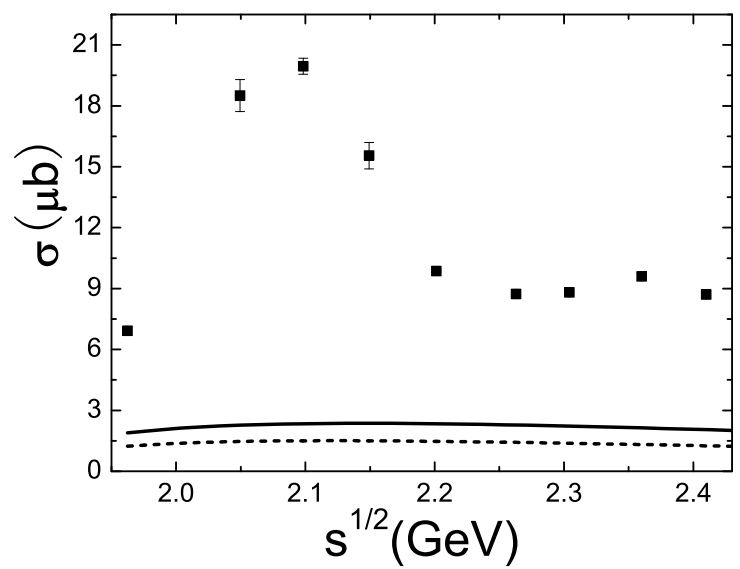

FIG. 3: Cross sections for $p \bar{p} \rightarrow \eta \eta$ with $\cos \theta$ integrated from 0 to 0.85 [18]. The dashed and solid curves show the contribution from the t-channel $N^{*}(1535)$ exchange with $\Lambda=3$ and $3.5 \mathrm{GeV}$, respectively.

In summary, we have phenomenologically studied the contribution of the t-channel $N^{*}(1535)$ resonance exchange to the $p \bar{p} \rightarrow \phi \phi$ and $\eta \eta$ reactions. While it plays only a minor role for the $p \bar{p} \rightarrow \eta \eta$ reaction, it gives a significant contribution to the $p \bar{p} \rightarrow \phi \phi$ reaction, especially for the energies above $2.3 \mathrm{GeV}$. Below $2.3 \mathrm{GeV}$, the s-channel resonances may still dominates through the strange meson or baryon loops. Before one tries to extract any definite information about the strangeness of the proton from this reaction as suggested by Ref.[10], one should take these additional mechanisms into account.

\section{Acknowledgments}

Useful discussions with Xu Cao, Jia-Jun Wu, Lu Zhao and Pu-Ze Gao are gratefully acknowledged. This work is supported by the National Natural Science Foundation of China (NSFC) under grants Nos. 10875133, 10821063, and by the Ministry of Science and Tech- 
nology of China (2009CB825200).

[1] S. Okubo, Phys. Lett. B 5, 165 (1963); G. Zweig, CERN Report No. 8419/TH412, 1964; J. Iizuku, Prog. Theor. Phys. Suppl. 38, 21 (1996).

[2] B. S. Zou, Phys. Atom. Nucl. 59, 1427 (1996).

[3] V.P. Nomokonov and M.G. Sapozhnikov, Phys. Part. Nucl. 34, 94 (2003).

[4] J. Ellis, E. Gabathuler and M. Karkiner, Phys. Lett. B 217173 (1989); C. Amsler et al., Crystal Barrel Collaboration, Phys. Lett. B 346, 363 (1995); M.G. Sapozhnikov, Nucl. Phys. A 655151 (1999).

[5] S. J. Lindenbaum, Nuovo Cim. A 65, 222 (1981).

[6] A. Etkin et al. , Phys. Lett. B 201, 568 (1988).

[7] C. B. Dover, P. M. Fishbane, Phys. Rev. Lett. 62, 2917 (1989).

[8] N. I. Kochelev, Phys. Atom. Nucl. 59,1643 (1996).

[9] J. R. Ellis, E. Gabathuler, M. Karliner, Phys. Lett. B 217, 173 (1989).

[10] J. R. Ellis, M. Karliner, D. E. Kharzeev, M. G. Sapozhnikov, Phys. Lett. B 353, 319 (1995); Nucl. Phys. A 673, 256 (2000).

[11] H. J. Lipkin, Nucl. Phys. B 244, 147 (1984); 291, 720 (1987).

[12] Y. Lu, B.S. Zou, M.P. Locher, Z. Phys. A 345, 207 (1993).

[13] V. Mull, K. Holinde, J. Speth, Phys. Lett. B 334, 295 (1994).

[14] J. J. Xie, B. S. Zou, H. C. Chiang, Phys. Rev. C 77, 015206 (2008).

[15] Xu Cao, J. J. Xie, B. S. Zou, H. S. Xu, Phys. Rev. C 80, 025203(2009).

[16] M. Doring, E. Oset and B. S. Zou, Phys. Rev. C 78, 025207 (2008).

[17] C. Evangelista et al. , JETSET Collaboration, Phys. Rev. D 57, 5370 (1998).

[18] A.V. Anisovich et al., Nucl. Phys. A 662, 344 (2000).

[19] A.V. Anisovich et al., Nucl. Phys. A 662, 319 (2000). 